\title{
Investigation of a Tessellated Meta-Material Planar Circuit
}

\author{
Kimberley W. Eccleston \\ Dept of Electrical and Computer Engineering, University of Canterbury, \\ Christchurch, New Zealand \\ Tel: +64-3-364 2987 ext 7045, Fax: +64-3-364 2761, \\ E-mail: kim.eccleston@canterbury.ac.nz
}

\begin{abstract}
In this paper we investigate the propagation behavior of a square shaped planar meta-material guiding structure comprised of lefthanded and righted handed square unit cells each of the same size and arranged in a checker-board tessellation. The size of the planar structure is 19 cells by 19 cells and each cell is $8 \mathrm{~mm}$ by $8 \mathrm{~mm}$. At 1 GHz, the left-handed unit cells have an insertion phase of $11^{\circ}$ and the right-handed unit cells have an insertion phase of $-11^{\circ}$. Both types of cells have the same Bloch impedance. The structure was fed at the centre unit cell, and there are a total of 76 peripheral ports each terminated in matched loads. At $1 \mathrm{GHz}$ the power to each peripheral is equal and the phases at each port alternate between two values that differ by $11^{\circ}$. Moreover, the planar structure exhibits the so-called infinite wavelength phenomenon in two dimensions and could be used in place of a circular radial power divider.
\end{abstract}

Index Terms - Meta-materials, left-handed materials, negative refractive index, planar circuits, power divider.

\section{INTRODUCTION}

Meta-materials having negative refractive index have attracted much attention to the microwave community [1] of which the realisation of a planar lens [2] is a well known application. Practical microwave applications, however, have been largely based upon one-dimensional (1D) lefthanded transmission lines [3]. An important meta-material microwave device is the composite left-handed (LH) / right-handed ( $\mathrm{RH})$ transmission lines [4] that yields zero degree phase shift over a finite non-zero distance [5]-[7] - the so called infinite wavelength phenomena [7]. These devices comprise alternating $\mathrm{LH}$ and $\mathrm{RH}$ unit cells with equal but opposite insertion phase. There exists theory for two-dimensional (2D) meta-materials constructed of periodically loaded transmission lines of use to microwave engineering [8]. Twodimensional meta-materials structures that have been demonstrated usually comprise distinct $\mathrm{LH}$ and RH regions comprising many unit cells [9][10]. In this work we investigate a planar mosaic of $\mathrm{LH}$ and $\mathrm{RH}$ unit cells arranged in a checker-board tessellation. We show that such a structure exhibits the infinite wavelength phenomenon in $2 \mathrm{D}$ and could be used in place of a circular radial power divider.

\section{ANALYSIS OF THE MOSAIC}

Fig 1 depicts a 3 by 3 checker-board tessellation (or mosaic) of LH and RH unit cells. Essentially, all cells are 4-port elements with identical Bloch impedances. The insertion phase is $\varphi$ for a $\mathrm{LH}$ cell and $-\varphi$ for a RH cell. The central cell has a fifth port and the insertion phase from this port to any of its periphery ports is $-\varphi / 2$. The peripheral ports of the entire structure are terminated in matched impedances to form a non-reflecting boundary. The impedance of these terminations is the unit cell Bloch impedance.

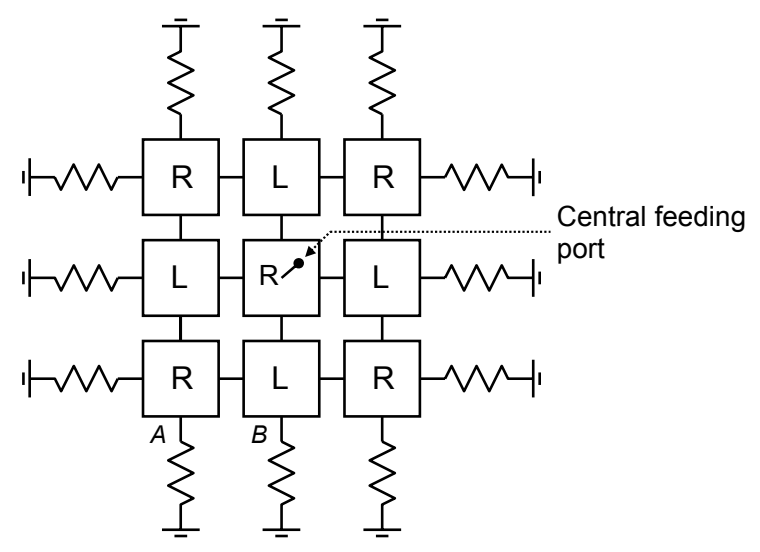

Fig. 1. A 3 by 3 LH RH mosaic.

Consider a wave incident on the central port. It will emerge from the peripheral ports of the central cell and be incident on all four LH cells. From each LH cell, the emerging waves are incident into a non-reflecting load and two corner located RH cells and so forth. By examining all the possible unique signal paths from the central feeding port to a periphery port, we see that the insertion phase is $-\varphi / 2$ from the central port to peripheral port $A$, and $\varphi / 2$ from the central port to peripheral port B. Further power is equally distributed among all peripheral terminations. Because the unit cells are lossless, the input impedance of the central port is $Z_{B} / 12$, where $Z_{B}$ is 
the Bloch impedance, and the coupling between the central port and the peripheral port is 0.289 . These outcomes have been confirmed by circuit simulation. These concepts can be extended to larger mosaics and we restrict ourselves to square mosaics with odd numbered dimensions. For an $\mathrm{N}$ by $\mathrm{N}$ mosaic, the central port input impedance is $Z_{B} / 4 \mathrm{~N}$ and the coupling between the central and peripheral ports is $1 / 2 \sqrt{\mathrm{N}}$.

\section{SimulATION OF A 19 BY 19 MosAiC}

We now consider the simulation of a mosaic with $\mathrm{N}$ equal to 19 operating at $1 \mathrm{GHz}$. In this case there are 76 peripheral ports and one central port. The Bloch impedance was chosen to be 100 $\Omega$. Fig 2 shows the topology of a RH cell which comprises four right-handed conventional transmission lines each with characteristic impedance $Z_{o}$ and length $d_{R}$ where $Z_{o}$ is equal to the Block impedance $Z_{\mathrm{B}}$. For argument sake, we assume that the conventional right-handed transmission lines are air-filled. Fig 3 shows the topology of a LH cell which comprises four capacitors $2 C$, an inductor $L$ and four lengths of right-handed transmission lines forming the host medium [8]. The conventional transmission lines within the LH cells have similar properties to that in the RH cells except that their lengths are $d_{L}$.

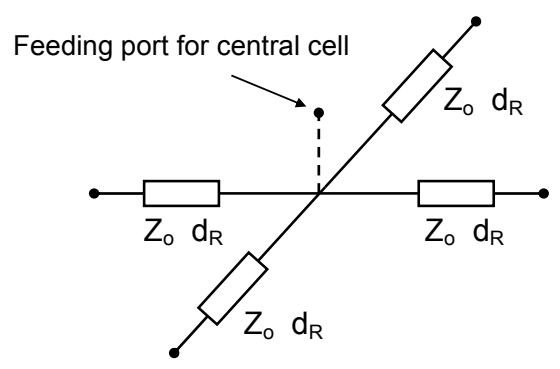

Fig. 2. Right-handed unit cell.

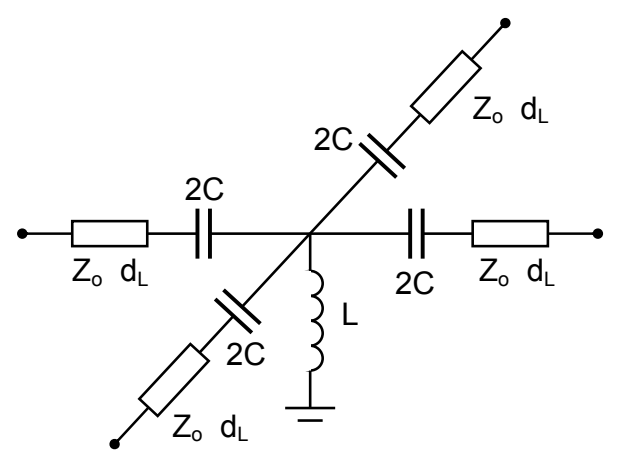

Fig. 3. Left-handed unit cell.
The insertion phase between two peripheral ports of a RH cell is:

$$
\varphi_{R}=-2 \beta d_{R}
$$

where $\beta$ is the phase constant of the conventional transmission lines. For the case of the LH cell:

$$
\varphi_{L}=\frac{1}{\omega \sqrt{L C}}-2 \beta d_{L}
$$

provided:

$$
Z_{o}=\sqrt{\frac{L}{C}}
$$

and the frequency is sufficiently high [5][8]. Clearly, the first term of (2) must have a magnitude greater than the second term in order to show left-handedness. If $C=4.37 \mathrm{pF}$ and $L=$ $43.7 \mathrm{nH}$, then (3) gives $100 \Omega$, and the first term of (2) corresponds to $20.9^{\circ}$. With $d_{\mathrm{L}}$ equal to 4 $\mathrm{mm}$, the insertion phase of the LH cell (2) is $11.3^{\circ}$. Using (1), $d_{\mathrm{R}}$ needs to be $4.7 \mathrm{~mm}$ to give an insertion phase of $-11.3^{\circ}$. Meandering of the transmission lines could be used to ensure that the $\mathrm{RH}$ cells are the same physical size as the LH cells. The component values are reasonable for a $1 \mathrm{GHz}$ operating frequency and are comparable to other reported investigations using discrete components [5][8].

Fig 4 shows the S-parameters of a 1D version of the LH cell. The S-parameter reference impedance is $100 \Omega$. The results show that the insertion phase is leading, and is matched to 100 $\Omega$, at frequencies around $1 \mathrm{GHz}$. The simulated insertion phase is $11.3^{\circ}$ at $1 \mathrm{GHz}$. Being a composite $\mathrm{RH}$ and $\mathrm{LH}$ structure, the $\mathrm{LH}$ unit cells become right-handed above $1.5 \mathrm{GHz}$.

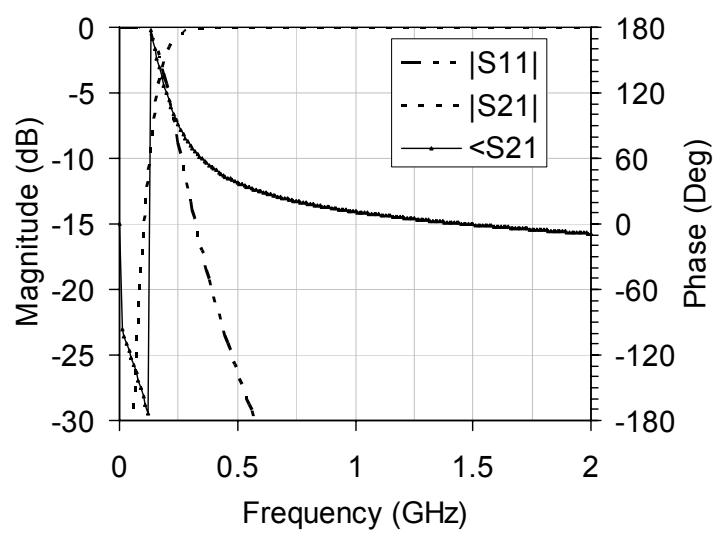

Fig. 4. S-parameters of $1 \mathrm{D}$ version of an LH unit cell. The reference impedance is $100 \Omega$.

The 19 by 19 mosaic was simulated using a commercially available microwave circuit simulator. The ports were numbered such that port 1 is the central feed port, and the peripheral ports are numbered from 2 onwards starting from a corner port. The planar circuit contains 361 unit 
cells, 1 central feeding port and 76 peripheral ports. During the simulations, owing to symmetry, only 10 of these peripheral terminations were designated ports (Ports 2 to 11 ). The reference impedance for the central ports was set to $1.32 \Omega$ (being $100 / 76$ ), and the reference impedance for the peripheral ports are $100 \Omega$. Altogether, the circuit to be simulated comprises: 1441 transmission lines, 180 inductances, 720 capacitances, 77 terminations (either resistances or defined ports). Therefore, simulation time is not negligible.

(a)

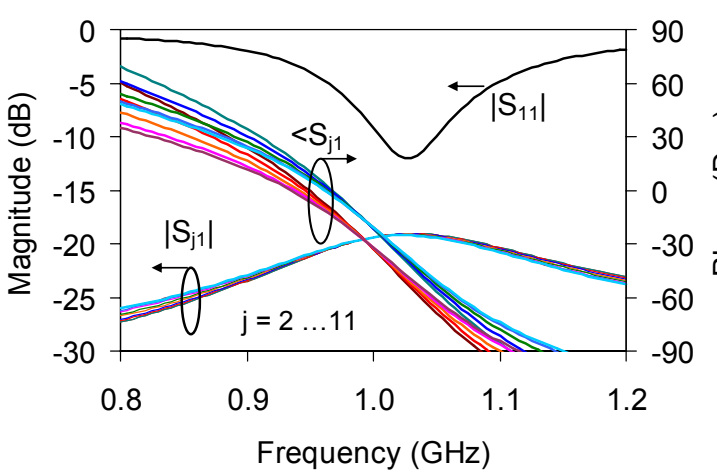

(b)

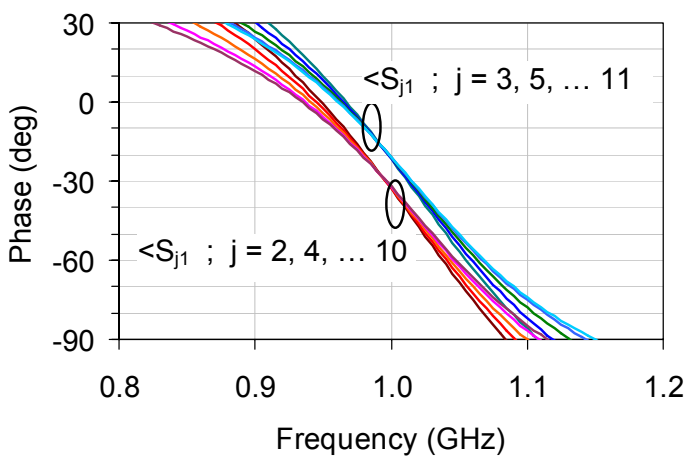

Fig. 5. S-parameters of 19 by 19 mosaic: (a) all relevant S-parameters, and (b) phase angles of $\mathrm{Sj} 1$ shown for clarity.

Fig 5 shows the simulation results of the 19 by 19 mosaic of $\mathrm{LH}$ and RH cells. These results show that the structure essentially works as theoretically expected. Namely at $1 \mathrm{GHz}$, port 1 has good return loss, equal power division with phases alternating between two values that differ by $11.7^{\circ}$. The central feeding port reference impedance was adjusted but best performance was obtained when equal to $1.32 \Omega$. The coupling between the central and peripheral ports is -19.4 $\mathrm{dB}$ which compares well with a theoretical value of $-18.8 \mathrm{~dB}$. The deviation from theory is combined effects of mismatch (albeit very low) of the LH unit cells. It is interesting to note that the group delays (slope of phase response) of the transmissions $\mathrm{S}_{\mathrm{j} 1}(\mathrm{j}=2 \ldots 11)$ are similar over a bandwidth of at least $20 \%$.

As a comparison, it is useful to consider a mosaic comprising of entirely RH cells. Such a structure is not unlike a square section of discretized parallel plate waveguide fed at its centre. Fig 6 shows the simulation results, which as expected, show significant phase variation and group delay for $S_{j 1}$ (for $\left.j=2 \ldots 11\right)$. Fig 6 shows the best central port match that could be obtained by varying its reference impedance.

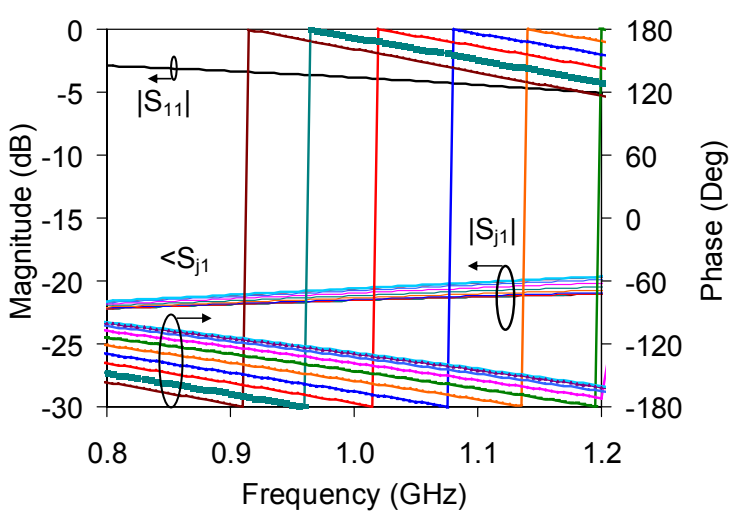

Fig. 6. S-parameters of 19 by 19 mosaic comprised of only RH cells. Reference impedance of central feeding port is $12.8 \Omega$.

\section{SPATIAL BEHAVIOR}

The results up to this point have been focused upon the interaction between the central and peripheral ports. To gain some insight into the behavior of the mosaic structure, it is of interest to consider the spatial variation of voltages across the planar structure.

The simulations were repeated but voltage probes were inserted at the centre of each unit cell. There were therefore a total of 361 probes for the 19 by 19 mosaic. Simulation was performed at 1 $\mathrm{GHz}$ only and the available power from the generator connected at the central feeding port was $0 \mathrm{dBm}$. The voltage magnitude and phase for each probe was collected for presentation using graphics software.

It was found that the voltage magnitude across the mosaic was between 44.4 and $48.8 \mathrm{mV}$, or 0.8 $\mathrm{dB}$ and the phase was between $-27^{\circ}$ and $-20^{\circ}$. Or in other words, the voltage across the mosaic is nearly constant. Fig 7 shows the spatial variation of the voltage across the entire mosaic. The contours for the Fig 7(b) are in steps of $0.5^{\circ}$. The conclusion here is that the mosaic exhibits the so called infinite wavelength phenomenon in 2D.

For comparison, the simulation was repeated for the case of a 19 by 19 mosaic comprised of 
entirely RH unit cells and the results are shown in Fig 8. In this case, as expected, the magnitude and phase variation is significant. The magnitude varies by $13.9 \mathrm{~dB}$, and phase varies $71^{\circ}$ across the side of the mosaic. Moreover, the behavior is that expected of cylindrical waves.

(a)

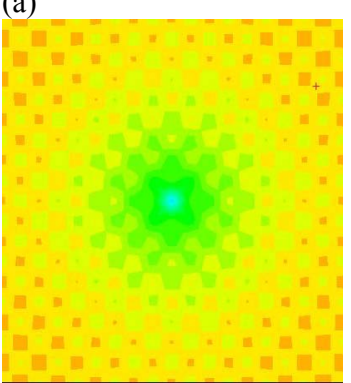

Fig. 7. Voltage across the mosaic at $1 \mathrm{GHz}$ : (a) magnitude with range $1.5 \mathrm{~dB}$, and (b). phase with contours in steps of $0.5^{\circ}$.
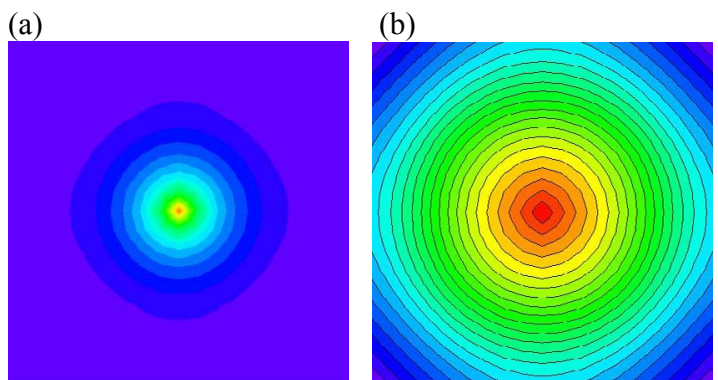

Fig 8. Voltage across the mosaic comprised of only $\mathrm{RH}$ cells at $1 \mathrm{GHz}$ : (a) magnitude with range $15 \mathrm{~dB}$, and (b) phase with contours in steps of $10^{\circ}$.

\section{CONCLUSION}

In this paper we have investigated a metamaterial planar circuit comprised of $\mathrm{LH}$ and $\mathrm{RH}$ unit cells arranged in a checker board tessellation of size 19 cells by 19 cells. At $1 \mathrm{GHz}$, the insertion phase was $11^{\circ}$ for each $\mathrm{LH}$ unit cell and $-11^{\circ}$ for each RH unit cell, and the Bloch impedance of all unit cells were identical. The structure was fed at its central node and the peripheral ports were terminated with matched terminations. The circuit demonstrated the so called infinite wavelength phenomenon in $2 \mathrm{D}$ at 1 $\mathrm{GHz}$. Moreover, power is equally divided to all peripheral terminations with phase alternating between two values that differ by only $11^{\circ}$. It is expected that the outcome would be unchanged for different sizes of mosaics provided that the number of rows and columns are identical and odd numbered.

With extra short compensating lines, it is conceivable that equal-phase power division is possible with a uniform planar structure with square sides. Or in other words, electrically equivalent to a circular shaped radial power divider but with straight sides and this makes it attractive for integration with amplifier modules (for example).

\section{REFERENCES}

[1] C. Caloz and T. Itoh, "Metamaterials for HighFrequency Electronics", Proc. of the IEEE, 93(10), pp 1744 - 1752, Oct 2005.

[2] J. B. Pendry, "Negative Refraction Makes a Perfect Lens", Physical Review Letters, 85(18), pp 3966 3969, 30 Oct. 2000.

[3] C. Caloz and T. Itoh, "Application of the transmission line theory of left-handed (LH) materials to the realization of a microstrip "LH" line", 2002 IEEE AP-S Int. Symp., pp 412 - 415.

[4] A. Sanada, C. Caloz and T. Itoh, "Characteristics of the Composite Right/Left-Handed Transmission Lines", IEEE Microwave and Wireless Component Letters, 14(2), pp 68 - 70, February 2004.

[5] M. A. Antoniades and G. V. Eleftheriades, "Compact Linear Lead/Lag Metamaterial Phase Shifters for Broadband Applications", IEEE Antennas and Wireless Propagation Letters, Vol. 2, pp 103 - 106, 2003.

[6] K. W. Eccleston, "Application of Left-Handed Media in Distributed Amplifiers", Microwave and Optical Tech. Letters, 44(6), pp 527 - 533, 20 March 2005.

[7] A. Lai, K. M. K. H. Leong and T. Itoh, "A Novel N-Port Series Divider Using Infinite Wavelength Phenomena", 2005 IEEE MTT-S Digest, pp 1001 1004.

[8] G. V. Eleftheriades, A. K. Iyer and P. C. Kremer, "Planar Negative Refractive Index Media Using Periodically L-C Loaded Transmission Lines", IEEE Trans. on Microwave Theory and Techniques, 50(12), pp 2702 - 2712, Dec. 2002.

[9] A. Sanada, C. Caloz and T. Itoh, "Planar Distributed Structures With Negative Refractive Index", IEEE Trans. on Microwave Theory and Techniques, 52(4), pp 1252 - 1263, April 2004.

[10] A. Lai, W-Y Wu, K. M. K. H. Leong, T. Itoh and C. Caloz, "Quasi-Optical Manipulations of Microwaves Using Metamaterial Interfaces”, 2005 IEEE AP-S Int. Symp., pp 273 - 276. 